\title{
"You've got to make it relevant": barriers and ways forward for assessing cognition in Aboriginal clients
}

Kylie M Dingwall ${ }^{1 *}$, Melissa A Lindeman ${ }^{2,3}$ and Sheree Cairney ${ }^{1,2,4}$

\begin{abstract}
Background: Reliable cognitive assessment for non-western cultures is difficult given that mainstream tests typically rely on western concepts, content and values. Despite recognition of the scarcity of appropriate tests for Aboriginal people over many years, limited practical development has occurred. This study aimed to identify barriers to assessment for clinicians working with Aboriginal people in a remote context, and evaluate characteristics of assessments that clinicians considered to be more or less appropriate and thereby identify potential ways forward.

Methods: Semi-structured interviews with 22 health professionals working with Aboriginal clients in central and northern Australia enquired about cognitive assessment practices for Aboriginal people. Themes related to common barriers, useful approaches and areas of need are reported.

Results: Results demonstrated that engagement, diversity and the lack of appropriate resources were seen as the most significant barriers to effective assessment. Appropriate tools should be visually engaging, brief, portable, relevant, focus on identifying strengths and cater to diversity whilst also being standardised and scientifically valid.

Conclusions: Despite the obvious challenges, further development is required to increase available resources and evidence base. Specifically, assessments suitable for younger populations and measuring substance misuse related impairments and change over time in a clinical setting were highlighted as important areas of need. A comprehensive assessment should include collection of data from multiple sources with clear interpretation guidelines.
\end{abstract}

Keywords: Cognitive assessment, Cross-cultural, Testing, Indigenous, Aboriginal

\section{Background}

Cognitive status for Aboriginal people has historically been very difficult to establish given the bias of mainstream cognitive assessment tools towards 'western' cultures (Davidson, 1995; Drew, 2000). For example, standardised cognitive tests are most often developed and interpreted within the scientific and cultural framework that dominates western or developed cultures (Dingwall \& Cairney, 2010). This typically emphasises the assessment of literacy and numeracy, and concepts such as prediction, problem solving and memory for world facts. Low English literacy, limited formal schooling, differing concepts of numbers, time and

\footnotetext{
* Correspondence: kylie.dingwall@menzies.edu.au

${ }^{1}$ Menzies School of Health Research, Institute of Advanced Studies, Charles

Darwin University, PO Box 4066, Alice Springs, NT 0870, Australia

Full list of author information is available at the end of the article
}

space, differing communication styles, and no tradition of written language in Indigenous cultures may therefore contribute to unreliable performance on such tests (Ardila, 2005; Janca \& Bullen, 2003; Kearins, 1988; LoGiudice et al., 2006; Westerman, 2004; Westerman \& Wettinger, 1997). These tests are consequently inappropriate for use with Aboriginal Australians for whom reduced performance can be taken to indicate cognitive impairment and subsequently lead to misdiagnosis.

Underestimation of 'true' ability can perpetuate myths and stereotypes which may in turn lead to increased marginalisation, discrimination and exclusion, particularly when tests are used for selection purposes (Drew, 2000). Unrecognised impairment may lead to labelling of clients as uncooperative, insolent or defiant and result in exclusion from rehabilitative programs for those most in need (Weinstein \& Shaffer, 1993). False positives in screening 
can lead to further assessment which may be distressing and wasteful of resources or lead to more serious consequences such as inappropriate prescriptions for medication (LoGiudice et al., 2006; Parker \& Philp, 2004). In short, tests have consequences and if error is increased, as is often the case in cross-cultural assessment, then adverse consequences are also increased (Goodnow, 1988).

In the absence of more appropriate tools, cognitive assessment for Aboriginal people has typically involved sustained use of mainstream scales, with or without modifications, qualifications or adequate evaluation (Dingwall \& Cairney, 2010; Dingwall, Pinkerton, \& Lindeman, 2013). While the inadequacy of cognitive assessment techniques for Aboriginal Australians has been recognised for a number of years, little practical development has occurred (Davidson, 1995; see Dingwall \& Cairney, 2010 for a complete review).

The Queensland Test, developed in the 1960s, was one of the first tests adapted specifically for use with Aboriginal people (Kearney, 1966; McElwain \& Kearney, 1970). Adapted versions of the Knox Cube Imitation Test, the Beads Test, Alexander's Passalong Test, the Form Assembly Test and the Pattern Matching Test constitute the five subtests. While administered using pantomime to minimise the effects of language, a relationship was observed between test performance and degree of contact with European or Western culture and the tasks are "still essentially Western in both origin and content" (Garton, 2003, p. 370). Three sets of norms for low, medium or high degrees of contact with western culture were developed, however these norms are now outdated (Drew, 2000) and no recent studies using this tool have been reported in the literature (Dingwall \& Cairney, 2010).

Since then, only one tool has been adapted and clinically validated specifically for use with Aboriginal Australians. The Kimberley Indigenous Cognitive Assessment (KICA) was developed to screen for dementia among Aboriginal people aged $\geq 45$ years from the Kimberley region of Australia (LoGiudice et al., 2006; Smith et al., 2007). It has since also been validated for use in the Northern Territory and North Queensland and adapted for use in other, nonremote regions in Australia (LoGiudice, Gibson, \& Savvas, 2013; LoGiudice et al., 2011; Marsh, Inglis, Smith, \& LoGiudice, 2007; Smith et al., 2009). As it has a specific purpose in dementia screening, it is not validated for assessing younger Aboriginal people with indications other than dementia, however it is sometimes used in the absence of other available validated tests (Dingwall et al., 2013).

More recently, computerised assessments such as Cambridge Neuropsychological Automated Test Battery (CANTAB) and CogState Ltd have been assessed for their utility in research with Aboriginal people (Cairney, Clough, Jaragba, \& Maruff, 2007; Cairney, Maruff, Burns, Currie, \& Currie, 2004, 2005; Dingwall, Lewis, Maruff, \&
Cairney, 2009, 2010; Lewis, Dingwall, Sawyers, Berkhout, \& Cairney, 2010). The strength of these assessments lies in their precision and sensitivity to change over time, but without relevant norms or cut scores for impairment and restricted accessibility, these tests are limited in their clinical applications.

These activities have been important but rare developments for cognitive assessment for Aboriginal Australians. It is therefore important to investigate the reasons for the limited progress in developing culturally relevant cognitive tests. The study aim was to identify perceived barriers to appropriate assessment and describe cognitive assessment formats considered more or less appropriate by clinicians in order to identify potential areas for further development.

\section{Methods}

Using qualitative enquiry, this study reviewed the current state of practice for assessing cognition among Aboriginal Australians. Purposive sampling with snowballing was used to recruit participants. Participants were primarily non-Aboriginal professionals who were experienced in conducting cognitive assessments for Aboriginal people, comprising 15 psychologists (including one Aboriginal psychologist), three occupational therapists (OTs), one speech pathologist, two aged care nurses, and one physician $(\mathrm{N}=22)$. Relevant experience in an Aboriginal context ranged from 6 months to over 20 years experience. A participant reference group comprising some participants, an additional OT and Aboriginal researcher was established, meeting at three time points, to guide the research process. The project was also reviewed by a Menzies School of Health Research (Menzies) Indigenous Reference Group for advice and guidance. Ethical approval was granted by the Human Research Ethics Committee for the NT Department of Health and Menzies (including an Aboriginal sub-committee) and the Central Australian Human Research Ethics Committee.

Written informed consent was obtained prior to conducting 18 individual or small group (2-3 people) semistructured interviews with participants either in person or over the telephone. The interviews took 45-60 minutes and consisted of the same stem questions used for all participants. Questions enquired about the practice context, reasons for assessment, current assessment methods, outcomes of assessments, and strategies for managing identified impairments. Interviews were audio-recorded and transcribed verbatim and data collection ended when data had reached saturation (i.e. no new themes emerging). Transcripts were critically analysed using thematic analysis assisted through NVivo software (Quinn Patton, 2002). Common themes were generated by organising interview data into a system of coded patterns, concepts and categories. Themes were identified inductively by author one, 
independently cross-checked with two co-researchers then discussed until consensus was reached. During the data collection and analysis phase, emerging themes were presented on two occasions to the participant reference group for verification and discussion. Pseudonyms are used to present results.

Selected themes relating to barriers to assessment, successful approaches and areas of developmental need are reported here. Themes relating to the range of tools in use and clinical practice guidelines for practitioners new to Aboriginal contexts has been published elsewhere (Dingwall et al., 2013).

\section{Results}

\section{Barriers to assessment}

\section{Engagement}

Difficulties in engaging Aboriginal clients in a meaningful way were seen as a significant barrier to assessment for clinicians. Social disengagement and disadvantage, mobility, and/or cognitive impairment itself were cited as contributing factors. Importantly, the mismatch between western and Aboriginal values and practices also contributed to poor engagement, borne out by a lack of perceived assessment importance, lack of motivation during assessments, and a historical mistrust of mainstream service providers.

"With our people here, they're people who are the poorest of the poor, incredibly disadvantaged... So it's pretty hard even to get them in here... they're not motivated to do their best necessarily. They might be tired, they might think 'what's this idiot whitefella talking to me about this [stuff] that doesn't make sense for?'... There are not many we see on an ongoing basis... and the ones who are engaged in therapy would probably not be impaired". (Chantelle)

"For the most part, the referral's coercive so the people haven't put their hands up and said "I would like to go and see [Neil] thanks for a cognitive assessment". So you know there's that aspect of resistance to have to work with as well". (Neil)

"But it's very hard because they move around so much. You can make an appointment to see someone and they won't show up, or they might show up three days later or three days earlier". (Robert)

\section{Time}

Time also impacted on ability to conduct cognitive assessments for Aboriginal clients, both because of the differing concept of time between Aboriginal and Western cultures, the perceived difficulties with maintaining of clients' attention and due to the limitations of 'efficient' service provision.
"So you've got a half an hour timeframe to get in there and get out while they're fresh, because unlike us, they're not used to sitting down for hours, because they like to move around and do different things or they quickly lose interest or [there's] a loss in motivation". (Robert)

Cognitive assessment was not always seen as a priority, as services often have different mandates and service foci. Some often did not have the resources or capacity to conduct comprehensive assessments, so there was reluctance to invest in resources given cognitive assessment was often just a small component of respondents' work.

\section{Diversity}

Another identified barrier was the high degree of diversity among Aboriginal culture, people and language, including regional differences in languages and beliefs, and diverse levels of contact with western practices, education, literacy and numeracy.

"But having worked the whole of the NT and big parts of $S A$ and WA, the people at Ramingining are incredibly different to the people at Areyonga, ... [or] Alice Springs. Their language is different, some of their beliefs are different, some of their traditions are different, so it's really really hard to come up with something that you think is suitable for 'Aboriginal People'”. (Kirsty)

Almost all cognitive tests are presented in English, so language is an important barrier, especially for a group of peoples for whom English can be a second, third, fourth or fifth language. Whilst some similarities exist between Aboriginal languages in particular regions, the diversity of different language groups presents further barriers in access to interpreters. Understandably, it can therefore be difficult to gauge whether the instructions for a test have even been understood.

Most respondents identified a clear difference between Aboriginal people who lived in urban or rural areas and those in remote regions given the diversity of contact with western practices and associated expectations. Choice of tests was therefore dependent upon the level of contact with western systems, primarily the education system.

"Obviously it's not just the language thing, it's also exposure to the majority culture, or the expectations of the majority culture as reflected in a standardised assessment". (Belinda)

"To test a little white kid like you or me who's been in school and does our homework over an hour and half that's one thing but to give some of these kids an hour and a half, well it's like purgatory, and after 
10 minutes, they're scratching and farting and carrying on, you know". (Martin)

"And if you know they don't know numbers and they don't know letters, well obviously there's nothing wrong with them, they simply don't know'. (Belinda)

Even if the client reports attending school, there is enormous diversity in the quality of the education received.

"Sometimes we'll even do on a whiteboard a timeline, just to get a sense of how often ..., how much they went to school. Some of the locals here go to boarding school in Adelaide, a fairly elite school, or was it in community homeland schools where English is a second language, not much attendance there..." (Felicity)

Identifying an individual's abilities compared to a 'normal' population is a difficult task given the extreme diversity within the Aboriginal population. Therefore, challenges arise in determining an appropriate normative sample for comparison.

"Yes, you have to try and contrast it against the norm which, again, is very difficult. Do you contrast it against a mainstream norm, a custodial norm, an Indigenous norm? It becomes quite sketchy”. (David)

\section{Limited resources, training and knowledge}

The lack of resources, evidence-base, normative data, training and knowledge regarding appropriate tools for Aboriginal people were identified as major barriers to effective assessment.

"But really there is a lack of what you can rely upon in doing cognitive assessment in this situation". (Scott)

It was also identified that mainstream approaches may not be applicable. In the absence of sufficient research, many clinicians are "working blind" (Natasha).

"Really it isn't ...good enough not to have the norms on these things. But on the other hand you can have Aboriginal norms developed in Queensland on the coast, and they wouldn't apply here. And it would be dangerous to say that they did. And even here compared to the Top End, it would be dangerous to use the same norms in terms of standing up and say this person functions on the x percentile". (Martin)

"We just do the best we can with what we have, so it's just adapting a lot of it... So we're using these, and its great here, because we then can adapt something, but are we adapting them appropriately? So we need more research on that". (Natasha)

Inappropriate testing formats for Aboriginal people included tests that were not engaging or interesting, language based tasks, purely question-answer formats, those that required a response within a specified time limit, and tasks that resembled school based tasks (e.g. pencil and paper tests) - particularly where individuals had not had positive schooling experiences.

"Time response doesn't work and 'look at these pictures and later on I'm going to ask you' and then ...

'remember what those pictures were?' But they'll talk about lots of other things and, you know, it just doesn't work and it's not a cognition thing, it's... I just don't think testing in that manner works, or wanting a response in that manner, they sort of seem to think that you're a bit crazy". (Lisa)

\section{Successful approaches}

"So I think you've got to have something that captures their attention". (Sarah)

Successful approaches identified were engaging, fun, non-threatening, intuitive, and visually appealing.

"Sometimes I do them and I think I don't even want to do - I'm not really interested in ... the results of the assessment. I'm interested in having something that I can work with, that I can sit on the other side of the table and get somebody talking to me". (Belinda)

Further, useful approaches involved plain English, a degree of performance based activity with role modelling and practice, and didn't rely extensively on language. It was also seen as useful to have tasks that were brief and portable so they could be delivered outside the office.

"[Something that] holds their attention, because the thing about this is, in the more traditional people, if they are cognitively impaired you can't give them a big battery to fill out". (Robert)

Most importantly, the tasks and stimuli needed to be relevant to the individual, tapping into their strengths and being localised to their particular region.

"If there was a "golden star" it would still have to be regionalised which is so hard. But there are similarities in Indigenous people all over Australia, but there are just so many differences". (Kirsty) 
"The problem is you've got to make it relevant". (Robert)

Focussing on strengths rather than deficits was seen as important. Highly visual approaches, such as drawing or pictures or use of computer-based tasks, were seen as successful methods of engagement and holding attention. Many clinicians also noted visuo-spatial ability as a strength for Aboriginal people who often conversely had low English literacy. Aboriginal people were also thought to respond better to concrete stimuli which visual formats facilitated.

"Always use visual stuff and you find once you start drawing things on the whiteboard people really focus on it. They pay attention much more than if you're just talking”. (Chantelle)

"[T]hey just tend to do better on visual. Remembering visual items for example. They just seem to do better on, and patterns and sequencing... For example... the Raven's Matrices... the Aboriginal people tend to follow the patterns, and see, "Oh yeah". They work out what's going on, better than most people". (Sampson)

The strengths based approach prioritised performance based tasks as it was understood that Aboriginal people performed well in response to role modelling, physical activity and having an opportunity for practice or to experience success. These tasks also generally relied upon very little language, being more intuitive, or instructions being communicated via gestures and minimal verbal input.

"Ravens, there is very little verbal instruction involved. You can do it using an interpreter. It's quite intuitive in many ways". (Scott)

Computer based tasks were therefore seen as a potentially useful approach given their highly visual, intuitive and interactive nature.

\section{"We've done some computer based stuff and that seems to go really well”. (Felicity)}

For gathering information from clients, successful approaches included having a 'yarn' rather than firing questions one after the other. Often, the first thing a person would do is tell clients about themselves and they would use stories and humour to engage and gather information.

"There's the circuitous way of talking about something and coming back to it like an elaborated story. It's seen as a very very appropriate way to do it rather than just skipping from one thing to another and rapid fire”. (Neil)
"Oh and asking the right questions, I guess that's probably the second thing, is about talking about the issues that are important to them, not the issues about what's important that we want to find out". (Michelle)

\section{Areas of need}

The Kimberley Indigenous Cognitive Assessment (KICA) was identified as a suitable dementia screen in older Aboriginal people. However, this highlighted a significant gap in what was available clinically for assessing functioning in younger age groups and for indications other than dementia such as for detecting substance-induced cognitive impairment. Apart from not being validated for younger populations, the KICA was thought not to assess the right functions for this group.

"It is around measuring the effect of alcoholism on somebody's cognitive status that we most get stuck on... Like maybe it is an early dementia, but maybe it isn't. Maybe it is just a general alcoholism-related impairment. And I'd much prefer to have something to use on those people than a KICA, because I don't think the dementia screen is the right thing for them". (Victoria)

The need for suitable cognitive assessments that could be utilised in a prison setting was also identified. This related to the ability to assess how well an individual would perform within a group program, what level of supports they might need before and after release, and to monitor any changes that might occur over time particularly in response to withdrawal from substances.

"What would be nice is to be able to track changes over time. So if someone comes in early on their term of imprisonment, if they've had a long history of... substance abuse. Whether there's a capacity to track... any of the changes as well as which areas are going to be... problematic so you can then again, pitch the intervention..." (Hayley)

When asked what functions participants would like to be able to test, concentration/attention, abstract reasoning, decision making, executive functioning, visuospatial functioning and adaptive functioning were all identified as important. Memory was particularly highlighted.

"If you're going to have any cognitive impairment I'd have to say about 80 per cent of people would have a flow on memory problem". (David)

Visual construction tests that require copying figures were identified as relevant and useful in an Aboriginal setting, particularly for detecting organic impairment. 
Delayed recall on this was also considered important to include.

"Memory for designs which is the one where you show them a geometric pattern for ten seconds then take it away and ask them to draw it. Now that is meant to be sensitive to alcohol impairment, according to the manual, and in my experience it is". (Martin)

Basic neurological measures including reaction time, movement and coordination tasks were also seen as potentially useful and less culturally biased.

"Well, finger to nose, thumb and circle, arm and leg. So, motor maturity, motor planning, fine control of the large and small muscles, kinaesthetic, body balance, symmetry of the left and right hand... So, it's really useful for that sort of thing, and it's quite fun to do". (Belinda)

Verbal abilities were seen as difficult to assess for Aboriginal people whose first language was not English, but thought to be an important aspect of functioning to consider, including tests of comprehension and praxis.

"Comprehension, some social comprehension I think is useful. And then some of that... electronic stuff, if you can mix that in". (Martin)

"I mean the degree of accuracy that many of my Indigenous clients have to draw the various shapes that measure praxis and things like this on, say the RUDAS or the MMSE, just absolutely beautiful. And yet other aspects, like I said are at times not that great, whereas in my non-Indigenous population, I find often praxis is not that terrific. But verbal stuff is ok". (Neil)

Essentially, it was considered important to use assessments that could determine whether a person was able to function adaptively in their daily life, make safe decisions, complete everyday activities, acquire new learning and apply new learning to new situations. For this reason, some participants stressed the importance of including a matrices component to assess abstract reasoning along with a measure of adaptive functioning. However more work was said to be required to develop more culturally relevant measures of adaptive functioning.

"Their ability to solve problems, to identify sequences, to identify disparities to solve problems through pattern analysis without using words or vocab or minimal input. So I would believe it would be crazy to not have one or other matrix based set of tests in there to give you that". (Martin)

"I think the research ... into developing adaptive functioning assessment tools for Indigenous communities... would be extremely valuable". (Uma)

Adapting tests using culturally appropriate stimuli such as wildlife was also seen to have potential.

"And I thought, what we could do is make a test of visual spatial memory functioning, which is pretty sensitive to the effects of alcohol related brain damage, hypoxic brain damage... using stimuli that's culturally, sits on the fence and is good in both cultures... So I thought what about using wildlife?" (Robert)

Tools that are easy to administer and easy to interpret are also needed.

"And the good thing about the Naglieri and the Ravens are, or if you think it's a good thing, you don't need to be a psychologist to administer them. [But] they assume that you have the ability to be able to interpret it". (Uma)

Respondents wanted more guidance in the interpretation of test scores away from numbers, dots and squares into the ways the scores reflected areas of impairment and how they might impact upon people's lives or the types of interventions that might be useful to assist the client.

"So if someone is high on this and low on that, what is that likely to mean?... And I guess it does need to have some ranges in it because it is never going to be $x=y$.

But an idea that, somewhere in this range, you can expect this sort of stuff. And these are other things to consider when you are making a case formulation. This person here might be quite concrete in their thinking so in interventions consider $d a, d a, d a, d a$, something like that,... Yeah, so some understanding of what the interpretations would actually mean in practice and [in] what you are doing". (Hayley)

Respondents identified the need for instruments to assist with gathering a good background history and contextual information.

"What I would tend to push for is... interviews/ observation assessment tests filled in by the assessor which is corroborated by an interview with family. So, trying to come up with an average between the two". (David) 
Whilst the informant approach was considered useful, it brings some difficulties as demonstrated in the following example:

"...qualitative assessments of adaptive behaviour, which was done with a parent or the care givers, and also with people who knew them well. It would be really funny because it would be things like... 'how well does the child manage within the community?'.. The parents were completely biased one way or the other... and if you asked other people who knew the family, well it actually depended on whether they were having a big fight with them or something. So, it was really, really hard to get accurate information". (Uma)

However, many clinicians reported that a common approach is to assess client functioning through observations during the clinical interview.

"Well just in the course of providing therapy I think you can pick up on an awful lot of things like a person's working memory, their ability to make sense of what it is that they're saying or what you're saying, or how they're trying to put together their own words, their sense of orientation to time and place". (Neil)

\section{Discussion}

In order for clinicians to adequately assess Aboriginal clients, novel and culturally relevant assessments that measure the specific cognitive functions impacted by substance abuse or other acquired brain injury, using culturally appropriate approaches, are needed. Ultimately, the reason for the assessment and the implications for future management are important factors in shaping the assessment process and the tools that might be used to provide information about the client that is relevant and practically useful (Dingwall et al., 2013). In line with previous crosscultural assessment research, the current findings suggest that test selection or future test development for Aboriginal clients should also be informed by the considerations in Table 1 and focus on aspects of the content, format and procedures, as well as the testing environment (Dingwall \& Cairney, 2010). Whilst characteristics of the tests themselves can be made more relevant and reflective of Aboriginal ontology, as is a key finding in this paper, a focus on cultural competency and cultural safety in preparing and supporting staff for conducting assessments cross culturally is also an important area for consideration, and addressed separately (Lindeman, Dingwall, \& Bell, In Press).

\section{The need for clinical tools}

The KICA is currently the only Indigenous-specific cognitive assessment widely available for clinical use (Dingwall \& Cairney, 2010; Dingwall et al., 2013; LoGiudice et al.,
Table 1 Considerations for test development or selection relevant for clinicians working with Aboriginal clients

\begin{tabular}{ll}
\hline Criteria & Example/justification \\
\hline Brief & Less than 30 minutes \\
Portable/mobile & $\begin{array}{l}\text { Delivery outside the office, playing } \\
\text { cards, drawing in the sand }\end{array}$
\end{tabular}

Easy to administer and interpret

Neuropsychologists not always available in remote regions, administration with interpreter or cultural consultant

Not timed

Accuracy or ability more important

Concrete, tangible, visual

Less reliance on abstract processes. Interactive stimuli that can be physically manipulated

No/plain/little language

Intuitive

Interpretable into client's first language

Fun/engaging

Little reliance on language

Game-like tasks, visually appealing

Performance based - role modelling

Practice

'Do' rather than 'say' tasks

Allows experience of success and learn by observation and doing

Repeatable/sensitive to change

Not reliant on question/ answer format

Not reliant on literacy, numeracy

Accessible

Multiple alternate forms

Yarning/storytelling preferable

Use ecologically relevant tasks

Ideally freely available and widely accessible

Standardised

But modifiable administration and interpretation to include contextual info

Utilise synchronous or simultaneous processing

Spatial relations, contextually presented information

Relevant and meaningful tasks Relationships, kinship mapping, yarning

Familiar and relevant stimuli Animals, relationships, bush foods

2013; LoGiudice et al., 2006; LoGiudice et al., 2011). Despite its evident utility as a dementia screen, it is reportedly not validated for use with people aged under 45 and does not examine the relevant functions for identifying substance misuse related impairment (Dingwall et al., 2013). Tests validated for younger populations and to measure substance misuse-related impairment and change over time were therefore identified as important areas of need for future development. Although previous research has examined the reliability of a computerised cognitive assessment for assessing substance misuse-related impairment (i.e. Cogstate Ltd) within a research paradigm (Dingwall \& Cairney, 2010; Dingwall et al., 2009, 2010; Lewis et al., 2010) this test presently has limited clinical application. Its strengths include standardised and quick administration, automated response recording, randomised presentation of stimuli, ability to assess a range of functions and record many responses in a short period, 
along with being engaging and repeatable (with multiple alternate forms). However, to date this tool has only been used for group comparisons in research (Dingwall et al., 2009). Its restricted accessibility and lack of Aboriginal norms or cut points for impairment limit its current clinical utility for individual screening or diagnosis. Whilst the Queensland Test was developed for and considered useful for minimising the effects of language or cultural differences, its norms are now considered outdated and it was not reported to be used at all by our participants (Dingwall et al., 2013; Drew, 2000). Nevertheless, the design of each of these tests offers some useful information for development of new or existing cognitive assessment tools.

\section{Design features of a clinical tool}

Our results suggest that clinicians consider that for Aboriginal clients in remote locations, tests should be highly visually appealing, intuitive, engaging and utilise concrete, relevant and familiar stimuli, with decreased reliance on language, literacy and numeracy. Previous literature also advocates reduced reliance on verbal content and scholastic skills (reading, writing, and arithmetic) when testing linguistically and culturally diverse groups (Carter et al., 2005; Goodnow, 1988; Wong, Strickland, Fletcher-Jansen, Ardila, \& Reynolds, 2000). With increasing usage in remote communities, tablet computers could be a useful administration format as they can be very visually and tactilely engaging, intuitive and interactive. Adapting instructions or language to the local context was considered important by practitioners in order to establish a reliable result. Utilising simple instructions, informal administration procedures, prompts and feedback have previously been suggested to help minimise misunderstanding of task requirements (Carter et al., 2005; Lopez, Lamar, \& ScullyDemartini, 1997). Utilising performance based tasks where the participant is required to "do" rather than "say" something (Greenfield, 1997; Kearney, 1966; Lopez et al., 1997; Wong et al., 2000), demonstrating the task, and utilising practice trials can also be especially important for Aboriginal Australians whose typical learning style involves observation and imitation (Department of Education and Children's Services, 1995).

Responses suggested that tests involving timed components can be unreliable, reflecting previous literature reporting that it can be difficult to make an Aboriginal person complete an unfamiliar task within a required timeframe, as they will often sit back and evaluate a task before doing it (Janca \& Bullen, 2003). Portability and brevity were considered important characteristics in developing or selecting tests for this population. It can be difficult to maintain motivation and attention for long periods and long assessment procedures can cause fatigue, stress or boredom (Teng \& Manly, 2005). This was thought to be particularly the case for remote Aboriginal clients who were not accustomed to sitting and attending for long periods, especially if they had not had this experience through formal schooling. The use of detailed scoring of a smaller number of items might help decrease test time as well as anxiety for the participant (Teng \& Manly, 2005). In order to reduce unfamiliarity and anxiety regarding the test situation, assessments are best performed in an environment familiar and comfortable for the participant, such as their own home or outdoors (Carter et al., 2005; Cleworth, Smith, \& Sealey, 2006; Dingwall et al., 2013).

The most important consideration however, was to use content, stimuli and formats that were relevant, familiar and engaging, such as pictures of wildlife or other local objects. Presentation of a test in a game-like format may reduce the impact of culture and may also help to reduce anxiety and maintain motivation (Sahakian \& Owen, 1992; Yeo Hong Huang, 2003). Kearins (1976) utilised a modified version of "Kim's Game" as a memory test and found equivalent or better performance among Aboriginal testtakers compared to their European counterparts. Most cultures have games so they provide a rich source of shared knowledge about the intended interactions. The use of game-like stimuli may encourage best performance, induce competitiveness and can be intrinsically motivating which are common characteristics required for valid neuropsychological assessment (Yeo Hong Huang, 2003). Davidson (1979) described the routine use of card games in Aboriginal populations and some clinicians in this study reported use of playing cards as informal assessment tools (Dingwall et al., 2013). Again, their success was dependent on a person's familiarity with playing cards. Given that engagement in assessment was identified as a key barrier to effective assessment for Aboriginal clients, the use of game-like tests might be particularly useful in this setting.

Tasks that focus on relationships rather than categories or lists were seen as more appropriate for an Aboriginal context. This reflects cross cultural literature that reports contextually organised material (e.g. memory for special arrangements) results in fewer cultural differences in memory performance than verbally loaded memory tests such as list learning (Rogoff \& Chavajay, 1995). Davidson (1979) suggested that when a task permits, Aboriginal people may be more likely to store and retrieve information in a simultaneous or synchronous manner (e.g., learning the value of card combinations), rather than a serial or successive manner (e.g., logical addition). In assessing cognitive capability for Aboriginal Australians, it is therefore important to consider both the familiarity of stimuli as well as the typical cognitive processes that may be used to interpret such stimuli. Indeed visuo-spatial abilities were considered by participants to be well developed among Aboriginal clients. Furthermore, the relevance of 
family relationships to Aboriginal groups suggest that kinship mapping or the construction of genograms might provide useful information on cognitive functioning for Aboriginal clients (Dingwall et al., 2013).

Importantly, as was the case with the development of the KICA (Smith et al., 2007), Aboriginal people themselves should be consulted to determine what are considered appropriate tasks and behaviours to include in assessing particular cognitive constructs, along with the most appropriate format for such tasks. Future research should involve consultation and ethnographic research with Aboriginal people to identify behaviours, tasks or formats that are considered appropriate within their specific cultures and reflect Aboriginal conceptualisations of the cognitive constructs to be assessed. Once the underlying constructs are defined and a set of tasks identified, a Delphi approach could be used to reach consensus on face validity, relevance and usefulness prior to empirical examination of validity and reliability.

Examining the validity of new tests empirically is, however, challenging. Approaches would vary depending on the specific condition or population being assessed. It is difficult to identify appropriate 'gold standard' tests for each condition, outside of a comprehensive clinical interview. This approach is only useful where clinical criteria already exist for diagnosing particular conditions, and thus cut points for impairment can be identified. An alternate approach involves the development of normative databases incorporating Aboriginal populations. Whilst this poses logistical, financial and regional challenges, particularly given the diversity between the different Aboriginal groups across Australia, it may be a useful way forward, that requires significant time and resource investment.

\section{Clinical tool as a component of an overall assessment}

Clinicians considered that perhaps the most important aspect of an assessment was contextualising findings "away from little dots" to the impacts for everyday functioning. Clinicians were clear that an assessment is only as useful as its interpretation and then what follows the assessment, and some suggested that any developed assessment should have clear guidelines for interpretation and explanations or examples for the implications of a particular result. This may be a reflection of the practice setting, in which cognitive assessment is not the mainstay of clinical work and although assessment is required, highly trained neuropsychologists are rare within the remote Australian workforce.

The real life relevance of cognitive tests have been questioned (Jorm, 1996). A comprehensive assessment system is needed that involves the use of supplementary qualitative information, or other measures, in addition to traditional testing instruments (Armour-Thomas \& Gopaul-McNicol, 1997; Dingwall et al., 2013; Gopaul-McNicol \& Clark-
Castro, 1997). The collection of contextual information is essential for any assessment. The KICA was identified as useful as it includes a component that does this reasonably well. As a direct questioning approach was considered inappropriate for Aboriginal groups, a novel approach might utilise story telling or 'yarning' to elicit relevant information.

Standardised behaviour rating scales for collecting self reported cognitive information could also be useful in this setting. Observation of everyday behaviour by staff, and reports from an informant, may have utility particularly where motivation and engagement in testing is low or longitudinal perspective is required (Jorm, 1996). Staff rating scales offer relevance in residential rehabilitation centres or other institutions with a potential carryover effect of modifying staff perceptions of client behaviour. For example, staff attitudes may shift from considering clients 'uncooperative' to greater understanding about impairment and care needs and subsequently reduce unrealistic expectations of clients (Jorm, 1996; Weinstein \& Shaffer, 1993). An area of need identified by clinicians in this study was the development of adaptive functioning measures that incorporate culturally relevant behaviours.

\section{Accompanying components and guidelines for a clinical assessment}

A comprehensive cognitive assessment should include information from various sources. It should also include indicators of daily functioning, as well as information on cultural, social and environmental context, presenting mental state, and cognitive capacity. Sources might include a cognitive test that uses relevant stimuli, is repeatable and sensitive to change over time, brief, portable, engaging, and performance based. They might also involve collection of relevant background history via self-report, informant-report and clinician-report scales for rating cognitive and adaptive functioning that address culturally relevant behaviours. A weighting system or guidelines for rating the fidelity of the information might assist in synthesising information, taking into consideration its perceived reliability. Further work is first required to develop more appropriate and relevant cognitive tests, and behaviour rating scales that reflect the behaviours and tasks that are traditionally important and relevant to Aboriginal people's lives in a format that is culturally acceptable and responsive.

\section{Limitations}

The paper was focussed on cognitive assessment in general and a range of clinician experiences were sought. As a result, the degree of each clinician's experience and training may have impacted on their perceptions and proficiency with particular tools. This information was not specifically collected. Furthermore, this paper assumes 
that some broad characteristics of test material and testing formats can be identified which may be more or less suitable or aligned with Aboriginal perspectives and experience. However, it is expected that the specific purpose of the test (e.g. screening or diagnosis) may impact the suitability of particular materials or formats. Additionally, the diversity of Aboriginal cultures may further limit the extent to which these suggestions apply. An important limitation of this study was that Aboriginal views were underrepresented as only one Aboriginal psychologist was available to be interviewed.

\section{Conclusions}

Results from this study demonstrated that engagement, diversity and the lack of appropriate resources, evidence base or normative data are the most significant barriers to effective assessment for clinicians working with Aboriginal clients. It is important to have tools that are engaging and cater to the diversity of Aboriginal people and their cultures whilst are also standardised and scientifically valid. Utilising locally relevant content, formats and approaches are useful additions to observing culturally appropriate engagement protocols and maintaining cultural safety in practice. Despite the considerable difficulties and required investment, further development is urgently required to increase the available resources and evidence base for appropriate cognitive assessment tools for use with Aboriginal people. Specifically, assessments suitable for younger populations and to measure substance misuse related impairment and change over time are important areas of need. Future development of a comprehensive assessment for Aboriginal people should involve input from Aboriginal people themselves, include collection of data from multiple sources including self report, informant report, standardised or informal cognitive tasks with strong visual and culturally relevant stimuli, social, medical and other history including substance use and recent changes in functioning along with clear guidelines for their use and interpretation as a whole.

\section{Abbreviations \\ CANTAB: Cambridge Neuropsychological Automated Test Battery; KICA: Kimberley Indigenous Cognitive Assessment; Menzies: Menzies School of Health Research; MMSE: Mini Mental State Examination; NT: Northern Territory; OT: Occupational therapist; RUDAS: Rowland Universal Dementia Assessment Scale; WAIS: Wechsler Adult Intelligence Scale.}

\section{Competing interests}

The authors declare that they have no competing interests.

\section{Authors' contributions}

KD conceived of and designed the study, carried out data collection, analysis and interpretation and prepared the draft manuscript. ML contributed to the research plan, data collection and analysis and critically revised the draft manuscript. SC provided critical appraisal and input into the draft manuscript. All authors read and approved the final manuscript.

\section{Acknowledgements}

The authors would like to sincerely thank the generous clinicians who contributed their time, expertise and knowledge to this project. Special thanks to Michael Tyrrell and Jennifer Pinkerton for their input into this project and to Stephen Ralph for his expert review of the draft manuscript as an Aboriginal forensic psychologist. This paper was supported by a Training Fellowship for Indigenous Australian Health Research from the National Health and Medical Research Council of Australia (NHMRC). The funding source had no input into the preparation of this manuscript and the views expressed in this publication are those of the authors and do not reflect the views of NHMRC.

\section{Author details}

'Menzies School of Health Research, Institute of Advanced Studies, Charles Darwin University, PO Box 4066, Alice Springs, NT 0870, Australia. ${ }^{2}$ Centre for Remote Health, Flinders University, Alice Springs, NT, Australia. ${ }^{3}$ Centre for Research Excellence in Rural and Remote Primary Health Care, Alice Springs, Australia. ${ }^{4}$ Cooperative Research Centre for Remote Economic Participation, Ninti One Ltd, Alice Springs, NT, Australia.

Received: 19 November 2013 Accepted: 23 May 2014

Published: 13 June 2014

\section{References}

Ardila, A. (2005). Cultural values underlying psychometric cognitive testing. Neuropsychology Review, 15(4), 185-195. doi:10.1007/s11065-005-9180-y.

Armour-Thomas, E, \& Gopaul-McNicol, S-A. (1997). Bio-ecological approach to cognitive assessment. Cultural Diversity and Mental Health, 3(2), 131-144. doi:1077-341 X/97/020131-14.

Cairney, S, Maruff, P, Burns, CB, Currie, J, \& Currie, BJ. (2004). Neurological and cognitive impairment associated with leaded gasoline encephalopathy. Drug and Alcohol Dependence, 73, 183-188. doi:10.1016/j.drugalcdep.2003.10.010.

Cairney, S, Maruff, P, Burns, CB, Currie, J, \& Currie, BJ. (2005). Neurological and cognitive recovery following abstinence from petrol sniffing. Neuropsychopharmacology, 30, 1019-1027. doi:10.1038/sj.npp.1300682.

Cairney, S, Clough, AR, Jaragba, MJ, \& Maruff, P. (2007). Cognitive impairment in aboriginal people with heavy episodic patterns of alcohol use. Addiction, 102, 909-915. doi:10.1111/j.1360-0443.2007.01840.x.

Carter, JA, Lees, JA, Murira, GM, Gona, J, Neville, BGR, \& Newton, CRJC. (2005). Issues in the development of cross-cultural assessments of speech and language for children. International Journal of Language and Communication Disorders, 40(4), 385-401. doi:10.1080/13682820500057301.

Cleworth, S, Smith, W, \& Sealey, R. (2006). Grief and courage in a river town: a pilot project in the aboriginal community of Kempsey, New South Wales. Australasian Psychiatry, 14(4), 390-394. doi:10.1080/j.1440-1665.2006.02310.x.

Davidson, G. (1979). An ethnographic psychology of aboriginal cognitive ability. Oceania, 4, 270-294.

Davidson, G. (1995). Cognitive assessment of indigenous Australians: towards a multiaxial model. Australian Psychologist, 30(1), 30-34. doi:10.1080/ 00050069508259601.

Department of Education and Children's Services. (1995). Aboriginal Culture and Maths Retrieved 30 May, 2008. from http://www.aboriginaleducation.sa.edu.au.

Dingwall, KM, \& Cairney, S. (2010). Psychological and cognitive assessment of indigenous Australians. Australian and New Zealand Journal of Psychiatry, 44(1), 20-30. doi:10.3109/00048670903393670.

Dingwall, KM, Lewis, MS, Maruff, P, \& Cairney, S. (2009). Reliability of repeated cognitive assessment in healthy indigenous Australian adolescents. Australian Psychologist, 44(1), 224-234. doi:10.1080/00050060903136839.

Dingwall, KM, Lewis, MS, Maruff, P, \& Cairney, S. (2010). Assessing cognition following petrol sniffing for indigenous Australians. Australian and New Zealand Journal of Psychiatry, 44(7), 631-639. doi:10.3109/00048671003627405.

Dingwall, KM, Pinkerton, J, \& Lindeman, MA. (2013). People like numbers: a descriptive study of cognitive assessment methods in clinical practice for aboriginal Australians in the Northern Territory. BMC Psychiatry, 13(42), 13. Retrieved from http://www.biomedcentral.com/1471-244X/13/42 doi:10.1186/1471-244X-13-42.

Drew, N. (2000). Psychological Testing With Indigenous People in Australia. In P Dudgeon, D Garvey, \& H Pickett (Eds.), Working with Indigenous Australians: a Handbook for Psychologists (pp. 325-332). Perth, WA: Gunada Press. 
Garton, A. (2003). Cognitive Development. In JP Keeves \& R Watanabe (Eds.) International Handbook of Educational Research in the Asia-Pacific Region (pp. 365-377). Dordrecht; London: Kluwer.

Goodnow, JJ. (1988). Issues and Changes in the Assessment of People from Minority Groups. In G Davidson (Ed.), Ethnicity and Cognitive Assessment: Australian Perspectives. Darwin: Darwin Institute of Technology.

Gopaul-McNicol, S-A, \& Clark-Castro, KB. (1997). Cognitive testing with culturally diverse children. Cultural Diversity and Mental Health, 3(2), 113-116. doi:1077-341X/97/020113-04.

Greenfield, PM. (1997). You can't take it with you: why ability assessments don't cross cultures. American Psychologist, 52(10), 1115-1124. doi:10.1037/0003066X.52.10.1115.

Janca, A, \& Bullen, C. (2003). The Aboriginal concept of time and its mental health implications. Australasian Psychiatry, 11(s1), S40-S44. doi:10.1046/ j.1038-5282.2003.02009.x.

Jorm, AF. (1996). Assessment of cognitive impairment and dementia using informant reports. Clinical Psychology Review, 16(1), 51-73. doi:10.1016/0272-7358(95)00056-9.

Kearins, JM. (1976). Skills of Desert Aboriginal Children. In GE Kearney \& DW McElwain (Eds.), Aboriginal Cognition: Retrospect and Prospect (pp. 199-212). New Jersey, USA: Humanities Press Inc.

Kearins, JM. (1988). Cultural Elements in Testing: The Test, the Tester and the Tested. In G Davidson (Ed.), Ethnicity and Cognitive Assessment: Australian Perspectives (pp. 60-70). Darwin: Darwin Institute of Technology Press.

Kearney, GE. (1966). Some Aspects of the General Cognitive Ability of Various Groups of Aboriginal Australians as Assessed by the Queensland Test. Brisbane: University of Queensland.

Lewis, MS, Dingwall, KM, Sawyers, S, Berkhout, N, \& Cairney, S. (2010). The assessment of cognition in an adolescent indigenous population. Australian Psychologist, 45(2), 123-131. doi:10.1080/00050060903352998.

Lindeman, MA, Dingwall, KM, \& Bell, D. Towards better preparation and support for health and social care practitioners conducting specialised assessments in remote Indigenous contexts. Australian Journal of Social Issues. In Press.

LoGiudice, D, Smith, K, Thomas, J, Lautenschlager, NT, Almeida, OP, Atkinson, D, \& Flicker, L. (2006). Kimberley indigenous cognitive assessment tool (KICA): development of a cognitive assessment tool for older indigenous Australians. International Psychogeriatrics, 18(02), 269-280. doi:10.1017/ S1041610205002681.

LoGiudice, D, Strivens, E, Smith, K, Stevenson, M, Atkinson, D, Dwyer, A, \& Flicker, L. (2011). The KICA screen: the psychometric properties of a shortened version of the KICA (Kimberley indigenous cognitive assessment). Australasian Journal on Aging, 30(4), 215-219. doi:10.1111/j.1741-6612.2010.00486.x.

LoGiudice, D, Gibson, S, \& Savvas, S. (2013). Indigenous Cognitive Assessment Modification and Validation of the KICA in Victoria. Phase 1. Victoria: Alzheimer's Australia.

Lopez, EC, Lamar, D, \& Scully-Demartini, D. (1997). The cognitive assessment of limited-english-proficient children: current problems and practical recommendations. Cultural Diversity and Mental Health, 3(2), 117-130. doi:10.1037/1099-9809.3.2.117.

Marsh, G, Inglis, M, Smith, K, \& LoGiudice, D. (2007). Validation of the Kimberley Indigenous Cognitive Assessment Tool (KICA) in Rural and Remote Indigenous Communities of the Northern Territory. Retrieved from http://www.fightdementia. org.au/commonfiles/NT/validation_of_the_Kimberley_indigenous_cognitive_ assessment_tool_(KICA).pdf.

McElwain, DW, \& Kearney, GE. (1970). Queensland Test Handbook. Melbourne: A.C.E.R.

Parker, C, \& Philp, I. (2004). Screening for cognitive impairment among older people in black and minority ethnic groups. Age and Aging, 33(5), 447-452. doi:10.1093/ageing/afh135.

Quinn Patton, M. (2002). Qualitative Research and Evaluation Methods (3rd ed.). California: Sage Publications Inc.

Rogoff, B, \& Chavajay, P. (1995). What's become of research on the cultural basis of cognitive development? American Psychologist, 50(10), 859-877. doi:10.1037/0003-066X.50.10.859.

Sahakian, BJ, \& Owen, AM. (1992). Computerized assessment in neuropsychiatry using CANTAB: discussion paper. Journal of the Royal Society of Medicine, 85, 399-402. doi:0141-0768/92/070899-04.

Smith, K, LoGiudice, D, Dwyer, A, Thomas, J, Flicker, L, Lautenschlager, NT, \& Atkinson, D. (2007). 'Ngana minyarti? What is this?' Development of cognitive questions for the Kimberley indigenous cognitive assessment. Australasian Journal on Aging, 26(3), 115-119. doi:10.1111/j.1741-6612.2007.00234.x.
Smith, K, Flicker, L, Dwyer, A, Marsh, G, Mahajani, S, Almeida, O, \& LoGiudice, D. (2009). Assessing cognitive impairment in indigenous Australians: re-evaluation of the Kimberley indigenous cognitive assessment in western Australia and the Northern Territory. Australian Psychologist, 44(1), 54-61. doi:10.1080/00050060802563463.

Teng, EL, \& Manly, JJ. (2005). Neuropsychological testing: helpful or harmful? Alzheimer Disease and Associated Disorders, 19(4), 267-271. doi:10.1097/01.wad.0000190805.13126.8e.

Weinstein, CS, \& Shaffer, HJ. (1993). Neurocognitive aspects of sustance abuse treatment: a psychotherapist's primer. Psychotherapy, 30(2), 317-333. doi:10.1037/0033-3204.30.2.317.

Westerman, T. (2004). Engagement of indigenous clients in mental health services: what role do cultural differences play? Australian e-Journal for the Advancement of Mental Health (AeJAMH), 3(3), 1-7. doi:10.5172/jamh.3.3.88.

Westerman, T \& Wettinger, M. (1997). Psychological Assessment and Intervention, Psychologically Speaking. Retrieved from http://www.indigenouspsychservices. com.au/publications.php accessed on 25 June 2014.

Wong, TM, Strickland, TL, Fletcher-Jansen, E, Ardila, A, \& Reynolds, CR. (2000). Theoretical and Practical Issues in the Neuropsychological Assessment and Treatment of Culturally Dissimilar Patients. In E Fletcher-Janzen, TL Strickland, \& CR Reynolds (Eds.), Handbook of Cross-Cultural Neuropsychology. New York: Kluwer Academic/Plenum Publishers.

Yeo Hong Huang, D. (2003). Testing Cognition with Playing Cards: A New Paradigm. Melbourne: University of Melbourne.

doi:10.1186/2050-7283-2-13

Cite this article as: Dingwall et al:: "You've got to make it relevant": barriers and ways forward for assessing cognition in Aboriginal clients. BMC Psychology 2014 2:13.

\section{Submit your next manuscript to BioMed Central and take full advantage of:}

- Convenient online submission

- Thorough peer review

- No space constraints or color figure charges

- Immediate publication on acceptance

- Inclusion in PubMed, CAS, Scopus and Google Scholar

- Research which is freely available for redistribution 